\section{THE USE OF INSTAGRAM FOR IMPROVING ACCESS TO APPLICATION GUIDANCE FOR LEVEL 1 PAEDIATRIC TRAINING}

Jai Radhika Thakrar, Shiv-Raj Sharma. UK

\subsection{6/bmjpo-2021-RCPCH.223}

Background It is known that there is room for improvement in recruitment to paediatric training. The current $\mathrm{RCPCH}$ application guidance is a 23 page document, and the RCPCH YouTube channel has $2 \times 1$ hour videos. Social media has proven to be a source of condensed, readily accessible information. Instagram is a social media platform used daily by millions. Instagram Stories can be used to post multiple videos/images in slideshow format, instantly reaching an interested audience. Use of such a platform has the potential to accelerate the distribution of paediatric application guidance in an intuitive, easily updatable format.

Objectives To investigate the suitability of Instagram to deliver application guidance for those interested in entering Level 1 Paediatric Training (ST1), and how this compares to popular platforms currently in use.

To survey how aware prospective applicants are of the official RCPCH application guidance.

To survey how prospective trainees would prefer to have their questions about applications addressed.

Methods A total of 4 minutes of short videos were produced by an ST1 paediatric trainee. The content was based on the document 'Application Guidance - Level 1 Paediatric Training (ST1)' by the RCPCH. These covered key elements of the application process including essential and desirable qualities, eligibility criteria, exams and interview format. These were posted via Instagram Stories to her Instagram page with a following of 11,500 people. $80.2 \%$ of followers were aged $18-$ 34 and $67.2 \%$ were female. Viewers were invited to complete an anonymous self-reported web survey.

Viewers were also invited to ask questions directly to the trainee using Instagram's 'Q\&A Box'. This allowed the trainee to answer anonymised individual questions, and share responses to the whole audience.

Results This series of Instagram Stories were watched by 2107 people within the first 24 hours. 134 survey responses were received after watching the Instagram Stories. $71.6 \%$ of respondents were from the UK, 64.9\% were medical students and $23.9 \%$ were junior doctors.

$100 \%$ of respondents reported that these Instagram Stories helped their understanding of the paediatric application process, of which $65.9 \%$ rated them $6 / 6$ for helpfulness.

$44.7 \%$ preferred receiving the application guidance in the format of Instagram Stories, compared with 32.6\% preferring official RCPCH documents. Instagram Stories were strongly preferred over YouTube videos $(59.7 \%)$, online seminars (54.5\%) and blogs (63.4\%).

$48.1 \%$ of respondents were not aware of the $\mathrm{RCPCH}$ Application Guidance.

82.2\% of respondents stated the Instagram 'Q\&A box' as their preferred method to have questions answered about the application process. Only $12.6 \%$ preferred corresponding with an email account.

Conclusions Instagram stories are a viable adjunct to provide application guidance to prospective applicants to paediatric training. Use of such a platform may be an excellent tool to provide more accessible information for medical students and junior doctors.
The Instagram 'Q\&A Box' provides an efficient way to address questions from prospective applicants, to a wide audience.

\section{A SNAPSHOT STUDY OF OBESITY, GENDER AND PUBERTY IN A MULTI-ETHNIC PAEDIATRIC POPULATION}

Shien Chen Lee, Nikitha Rajaraman, Justina Rweyemamu. UK

\subsection{6/bmjpo-2021-RCPCH.224}

Background Obesity is an emerging public health concern in the United Kingdom. The prevalence of overweight and obese children in Birmingham is higher than the national average in England $^{1}$. Royal College of Paediatrics and Child Health $(\mathrm{RCPCH})$ reported that 1 in 5 primary school children are obese or overweight, and this increases to 1 in 3 in secondary school.

During puberty, the body composition changes, but there is significant sexual dimorphism ${ }^{2}$.

Objectives To determine the prevalence of high BMI (Body Mass Index) in a multi-ethnic paediatric cohort and define its relationship with gender and pubertal phase.

Methods We collected data from General Outpatient Clinics and Paediatric Assessment Unit (PAU) between January - February 2020 and September - November 2020 respectively. Age, height $(\mathrm{m})$ and weight $(\mathrm{kg})$ for children between 5 and 16 years were recorded. Their BMI $\left(\mathrm{kg} / \mathrm{m}^{2}\right)$ were calculated and their corresponding BMI centiles determined using RCPCH Growth Charts.

The relationship between high BMI (overweight and obese) and puberty within each gender group was studied. The pubertal age cut-off was set as the mean age of onset of Tanner Stage 2: 10.5 years for girls and 11.1 years for boys ${ }^{3}$.

Results We obtained data from 260 patients (Outpatient: 104, PAU: 156). 32\% (84/260) of the children were overweight and obese (overweight: 12.3\%, 32/260) (obese: 20\%, 52/ 260). The number of overweight girls and boys were similar (girls: 6.5\%, 17/260) (boys: 5.8\%, 15/260); however there are more obese boys than girls (boys: 12\%, 30/260) (girls: $8 \%, 22 / 260)$.

The relationship between pubertal phase and high BMI was different for both genders such that more girls with high BMI were pubertal, and more prepubertal boys have high BMI. In the outpatient data, 26\% (8/30) pubertal girls and $30 \%$ (9/30) prepubertal boys were overweight or obese. In the PAU data, 31\% (17/54) overweight or obese children were pubertal girls and 30\% (16/54) were prepubertal boys.

There were 28\% (11/39) more girls with high BMI in the pubertal group compared to those in the prepubertal group, while 11\% (5/45) more boys with high BMI in the prepubertal group $(p=0.056$, Fisher's exact test). We accept that the sample size of this study is small, and more data collection is needed to see if we can obtain a statistically significant result.

Conclusions Our study shows that the relationship between pubertal phase and high BMI differs between boys and girl. Future studies in larger cohorts are needed to examine this relationship and develop effective interventions to tackle obesity during pubertal period. 